\title{
DESENVOLVIMENTO E AVALIAÇÃO SENSORIAL DO LICOR DE UVA CV. ISABEL
}

\author{
COELHO, Bruno Emanuel Souza ${ }^{1}$ \\ NÉZIO, Edsania Princelânia Xavier² \\ ARAÚJO, Andressa Alves de ${ }^{3}$ \\ COELHO, Carmem Lúcia ${ }^{3}$ \\ SOUSA, Karla dos Santos Melo de ${ }^{4}$ \\ BRAGA, Ana Carolina Dias ${ }^{5}$
}

RESUMO: A viticultura é uma atividade agrícola de grande importância social no Brasil. No Vale do São Francisco, esta atividade agrícola, tem apresentado grande expansão, e a cultivar 'Isabel' têm sido amplamente explorada na viticultura brasileira, destinada a indústria de sucos, doces, bebidas e vinagres, entretanto possui rápido amadurecimento e baixa resistência pós-colheita. A produção de licores constitui uma forma de contornar os problemas relacionados à comercialização de produtos perecíveis. O presente trabalho teve como objetivo elaborar um licor de uva, utilizando a cultivar 'Isabel', avaliar os atributos sensoriais (cor, aparência, odor, textura, sabor), avaliação global do produto e intenção de compra. O licor foi elaborado a partir da infusão da uva em aguardente com graduação alcoólica de $39^{\circ} \mathrm{GL}$, em proporção de 1:1, ou seja, para cada quilo de uva, foi adicionado um litro de álcool, deixado em repouso durante 10 dias, com adição de açúcar na forma de xarope. A avaliação sensorial foi feita utilizando uma escala hedônica de 9 pontos. Os resultados obtidos foram submetidos à análise descritiva. O licor de uva, elaborado a partir da cultivar 'Isabel', obteve uma excelente aceitabilidade sensorial pelos provadores, apresentando-se como uma opção agregar valor a uva, aumentando a diversidade de bebidas alcoólicas no mercado.

Palavras-chave: Vitis labrusca L..Bebidas alcóolicas. Processamento. Derivados de frutas.

\section{SENSORY DEVELOPMENT AND EVALUATION OF THE GRAPE LIQUID CV. ISABEL}

SUMMARY: Viticulture is an agricultural activity of great social importance in Brazil. In the São Francisco Valley, this agricultural activity has shown great expansion, and the 'Isabel' cultivar has been extensively exploited in the Brazilian viticulture, destined to the juice, sweet, drinks and vinegar industry, however it has fast maturation and low post- harvest. The production of liqueurs is a way of getting round the problems related to the marketing of perishable products. The objective of this work was to elaborate a grape liqueur, using 'Isabel' cultivar, to evaluate the sensory attributes (color, appearance, odor, texture, flavor), global product evaluation and purchase intention. The liquor was made from the infusion of grape in brandy with alcohol grade of $39^{\circ} \mathrm{GL}$, in a proportion of 1: 1, that is, for each kilogram of grape, one liter of alcohol was added, left for 10 days, with addition of sugar in the form of syrup. Sensory evaluation was done using a hedonic scale of 9 points. The results were submitted to descriptive analysis. The grape liqueur, made from the 'Isabel' cultivar, obtained an excellent sensorial acceptability by the tasters, presenting itself as an option to add value to the grape, increasing the diversity of alcoholic beverages in the market.

\footnotetext{
${ }^{1}$ Universidade Federal do Vale do São Francisco, Petrolina, Pernambuco, Brasil.

${ }^{2}$ Graduanda em Tecnologia em Alimentos, Instituto Federal de Educação Ciência e Tecnologia da Paraíba, campus Sousa.

${ }^{3}$ Graduanda em Tecnologia em Alimentos, Instituto Federal de Educação, Ciência e Tecnologia do Sertão

Pernambucano, campus Petrolina.

${ }^{4}$ Professora Adjunta, Colegiado de Engenharia Agronômica, Universidade Federal do Vale do São Francisco -

Campus Ciências Agrárias.

${ }^{5}$ Professora do Ensino Básico, Técnico e Tecnológico (EBTT), Instituto Federal de Educação, Ciência e Tecnologia do Sertão Pernambucano, campus Ouricuri.
} 
Keywords: Vitis labrusca L. Alcoholic beverages. Processing. Fruit derivatives.

\section{INTRODUÇÃO}

A uva é considerada uma excelente fonte de vitaminas e minerais, conhecida como um dos melhores agentes naturais para prevenção do câncer (SHAYANFAR; BODBODAK, 2014). Seus compostos bioativos e sua capacidade antioxidante atribuída aos flavonoides reduzem os radicais livres protegendo contra o estresse oxidativo e doenças crônicas, $\mathrm{O}$ consumo desta fruta e seus derivados garantem melhor qualidade de vida (CALDAS et al., 2015).

Os principais polos de viticultura tropical no Brasil são o Vale do Submédio São Francisco, o noroeste Paulista e o norte de Minas Gerais (ABREU et al., 2017). No Vale do São Francisco, esta atividade agrícola tem apresentado grande expansão, devido a fatores como luz, temperatura e disponibilidade de água que favorecem a produção desta cultura ao longo do ano (ALBUQUERQUE et al., 2013). As condições edafoclimáticas da região favorecem cinco ciclos de produção em 2 anos. No entanto, para equilibrar a produção com as lacunas do mercado, a região está produzindo dois ciclos por ano, no primeiro e no segundo semestre (ANDRADE et al., 2017).

A videira 'Isabel' é uma das principais cultivares de Vitis labrusca L., espécie originária do sul dos Estados Unidos e de onde foi difundida para outras regiões, destinada principalmente à indústria e apresenta normalmente cachos com tamanho e peso inferiores quando comparada a outras cultivares, tais como Itália e Benitaka, no entanto, mostra-se com maiores valores de sólidos solúveis (MASCARENHAS et al., 2010).

Por ser uma cultivar de uva tinta, muito rústica e fértil, a cv. Isabel proporciona colheitas abundantes com poucas intervenções de manejo, se adapta a diferentes usos, como: uva de mesa; na elaboração de vinhos branco, rosado e tinto, os quais, muitas vezes, são utilizados para a destilação ou na elaboração de vinagre; origina suco de boa qualidade; pode ser matéria-prima para o fabrico de doces e geleias (CAMARGO et al., 2010).

Apesar da rusticidade da uva de mesa 'Isabel', a cultivar possui cachos com bagas bastante aglomeradas, rápido amadurecimento e baixa resistência pós-colheita, cujas principais causas de perdas reportadas pelos produtores estão relacionadas à excessiva degrana, escurecimento do engaço, incidência de podridões, perda de massa e amolecimento das bagas, além de problemas relacionados às embalagens, ao manuseio e ao transporte (SILVA et al., 2012; SILVA et al., 2015).

Segundo Vieira et al. (2010), o licor é o produto obtido pela mistura de álcool, água, açúcar e substâncias que lhe fornecem aroma e sabor, em medidas adequadas, sem que haja fermentação durante sua elaboração. E por ser considerada bebida alcoólica digestiva, os licores são geralmente consumidos após as refeições, tais como, o jantar (SEBRAE, 2012).

A produção de licores constitui uma forma de contornar os problemas relacionados à comercialização de produtos perecíveis e aqueles que possuem aspectos visuais e formas inferiores aos exigidos pelo mercado de frutas frescas, mas que se encontra em bom estado de conservação e com excelente valor sensorial e nutricional. Além disso, é uma alternativa para enfrentar os problemas relacionados ao excesso de produção e baixos preços praticados em alguns períodos (TEIXEIRA, 2010).

Além disso, a produção de licores permite a obtenção de um produto rico em compostos fenólicos, proveniente dos frutos utilizados. Ademais, a cultura dos pequenos centros e áreas rurais, bem como, a flora regional e a disponibilidade de árvores e frutas nativas, influenciam no consumo de licores artesanais, aumentando a competitividade com produtos industrializados (OLIVEIRA et al., 
2015; SEBRAE, 2012; ALMEIDA et al., 2012).

Neste contexto, a elaboração de licores torna-se uma possibilidade para o aproveitamento dos frutos, agregando valor à produção e possibilitando o aumento na renda de pequenos agricultores, haja vista que seu processamento exige tecnologia simples e o produto final é comercializado em temperatura ambiente evitando, assim, custos com a cadeia do frio (BARROS et al., 2008; OLIVEIRA et al., 2015).

Uma grande variedade de frutas tem sido utilizada no processamento de licores, a exemplos do abacaxi (TEIXEIRA et al., 2012), tangerina (ALMEIDA et al., 2012), açaí (OLIVEIRA; SANTOS, 2011), maracujá (DIAS et al., 2011), camu-camu (VIEIRA et al., 2010), graviola (OLIVEIRA et al., 2014), entre outras, entretanto, são escassos os trabalhos de processamento de licores de uva.

Considerando-se a necessidade de estudos que explorem o potencial mercadológico de frutas, lhe agregando valor, dessa forma, objetivou-se utilizar a uva cv. Isabel como matéria-prima para a produção de licor e avaliar os atributos sensoriais, avaliação global do produto, e intenção de compra.

\section{MATERIAL E MÉTODO}

\section{Matéria-prima}

As uvas cv. Isabel utilizadas para elaboração do licor foram adquiridas junto aos médios produtores do município de Lagoa Grande - PE, situado na região do Vale do São Francisco. O clima da região é classificado como semiárido (BSh) de acordo com a classificação climatológica de Köppen e Geiger, com temperatura média de $24,8{ }^{\circ} \mathrm{C}$ e pluviosidade média anual de $585 \mathrm{~mm}$, com chuvas concentradas no verão (BECK et al., 2005).

Além das bagas, utilizou-se uma fonte de teor alcoólico, sendo uma aguardente destilada com graduação alcoólica de $39^{\circ} \mathrm{GL}$, e açúcar orgânico convencional em forma de cristal, para confecção do xarope. Estes itens foram adquiridos em comércio local do município de Petrolina-PE.

\section{Elaboração do licor}

O licor foi feito de forma artesanal. As uvas foram lavadas e sanitizadas em solução clorada a 30 ppm durante 15 minutos, após isso as uvas foram lavadas novamente em água corrente para retirada do excesso de cloro. Posteriormente as uvas foram desengaçadas, maceradas e colocadas em infusão com a aguardente em recipientes de polietileno previamente higienizados e sanificados com álcool á $70 \%$. A proporção foi de 1:1, ou seja, para cada quilo de uva, foi adicionado um litro de álcool. A infusão aconteceu durante 10 dias, em temperatura ambiente.

Após a infusão, foi feito uma filtragem e adicionado o xarope de açúcar cristal.Para o preparo do xarope, a partir de açúcar e água potável, foi 2 (duas) partes de açúcar para 1 (uma) parte de água e concentrou até obter uma calda a $65^{\circ}$ Brix. Utilizou-se cerca de 250 gramas de xarope para cada litro de licor primário.

Por fim, o licor foi envasado em recipientes de vidros, previamente esterilizados em água fervente durante 15 minutos, e armazenados sob refrigeração $\left(4^{\circ} \mathrm{C}\right)$.

\section{Avaliação sensorial do produto}

A análise sensorial do licor elaborado com uvas Isabel foi realizada simultaneamente durante a XI Jornada de Iniciação Científica e Extensão do IF Sertão-PE, no Pátio da Escola Estadual de Referência Fernando Bezerra - Ouricuri/PE, constituindo-se de 56 provadores, não treinados, saudáveis, maiores de 18 anos, e que estavam na instituição no momento da pesquisa, de ambos os sexos com vínculo a instituição. 
Aproximadamente $50 \mathrm{~mL}$ do produto foram servidos em temperatura ambiente $\left(25^{\circ} \mathrm{C}\right)$ em copos plásticos descartáveis, acompanhados de água mineral para limpeza do palato, e os participantes foram direcionados a uma cabine de papelão.

O processo foi realizado seguindo as normas e recomendações da ABNT (1998), e os testes utilizados na pesquisa foram: afetivo com escala hedônica e escala de atitude ou de intenção de compra, adaptado por Dutcosky (2011).

O teste da escala hedônica de aceitação, expressa o grau de gostar ou de desgostar de um produto. A escala utilizada neste teste: foi a de 9 (nove) pontos em que, em escala decrescente: (9) gostei extremamente; (8) gostei moderadamente; (7) gostei regularmente; (6) gostei ligeiramente; (5) não gostei, nem desgostei; (4) desgostei ligeiramente; (3) desgostei regularmente; (2) desgostei moderadamente e (1) desgostei extremamente, para avaliar a cor, aparência, textura, sabor, aroma e o produto em geral (Figura 1).

E para o teste afetivo de escala de atitude ou de intenção de compra, que expressa a vontade do avaliador de consumir, adquirir ou comprar, um produto que lhe é oferecido, utilizou-se a escala verbal de 5 (cinco) pontos, em escala decrescente: (5) certamente compraria; (4) provavelmente compraria; (3) talvez compraria/talvez não compraria; (2) provavelmente não compraria; (1) certamente não compraria (Figura 1).

Figura 1. Ficha de avaliação sensorial utilizada para avaliar a aceitação sensorial e a intenção de compra do licor de uva elaborado.

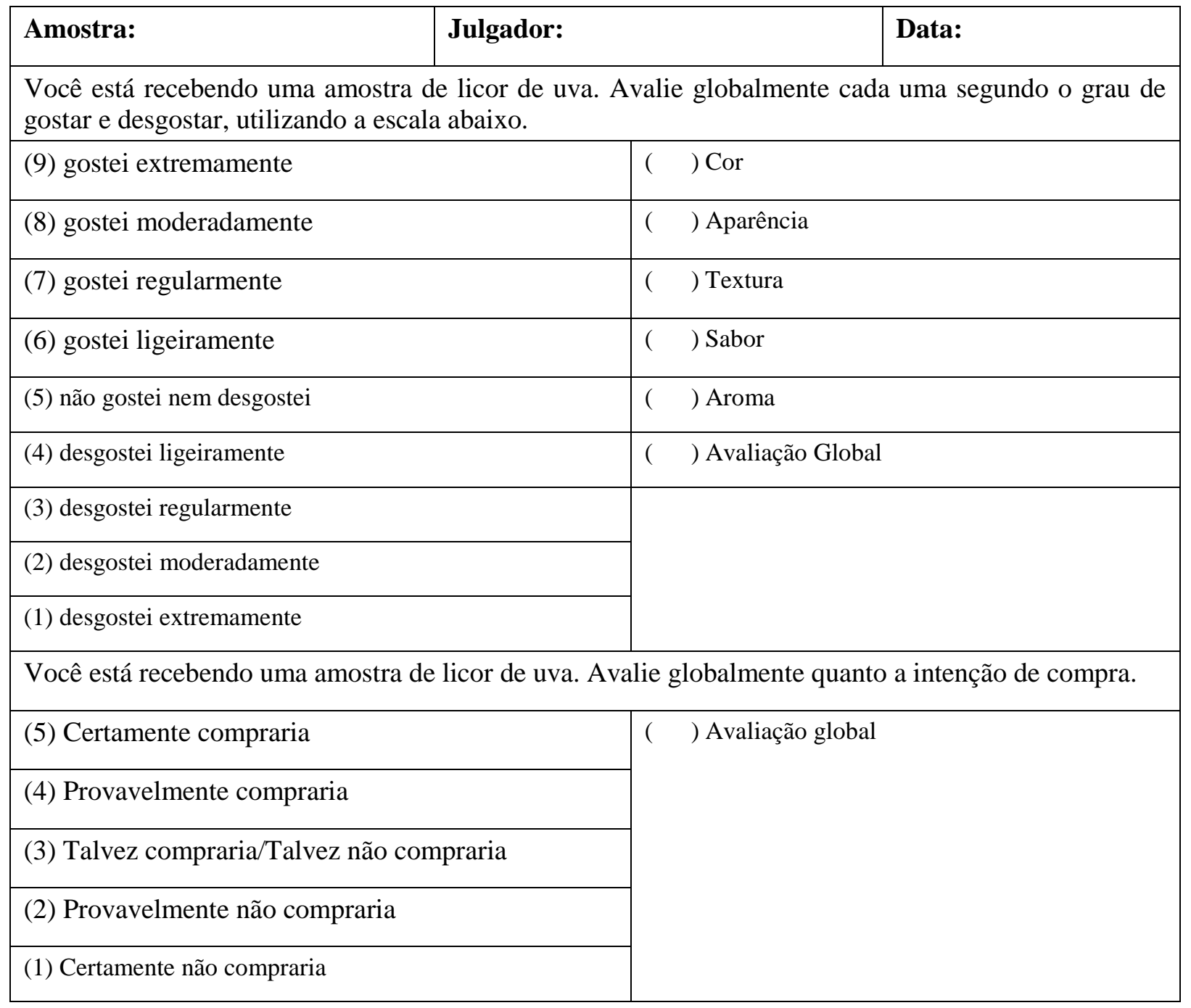




\section{Comentários:}

\section{Análise dos dados}

Os dados foram transformados em porcentagem e plotados em forma de gráficos, e calculado a média de todos os índices avaliados utilizado o software Sigmaplot 11.0.

Adicionalmente, foi determinado o índice de aceitabilidade (Equação 1) para cada atributo avaliado, a fim de verificar o grau de aceitabilidade do produto pelos consumidores Gularte (2009).

$I A=\frac{M}{N} * 100$

1)

Em que: IA: Índice de aceitabilidade sensorial (\%); M: média dos resultados dos julgadores; e N: número de pontos utilizados na escala de avaliação.

\section{RESULTADO E DISCUSSÃO}

A variável odor foi a que apresentou menor frequência de respostas "Gostei extremamente" e "Gostei moderadamente", com percentual de $85 \%$, e a média de todas as variáveis da frequência desta resposta foi de $89,93 \%$ (Figura 2).

Figura 2. Resultado da avaliação das variáveis sensoriais do licor de uva elaborado.

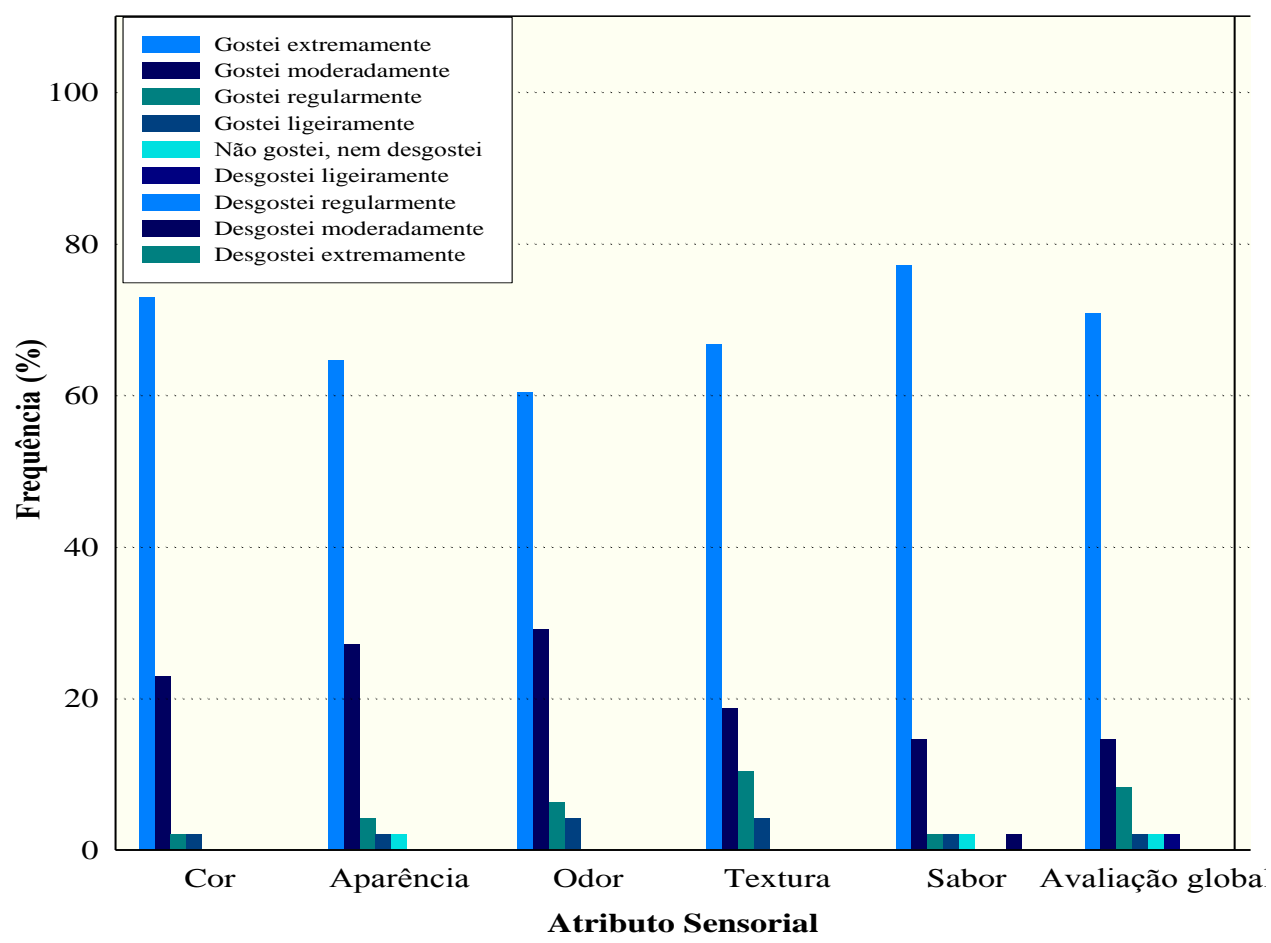

Nucleus,v.16,n.2,out.2019 
De modo geral as variáveis cor e sabor foram as que mais chamaram a atenção dos provadores sendo caracterizadas com maior frequência da nota 9,0 (gostei extremamente).

Por tanto, de acordo com Dutcosky (2011) e Gularte (2009), os atributos sensoriais do produto, superaram as expectativas do público, uma vez que uma frequência de $70 \%$ de respostas situadas entre o "Gostei extremamente", "Gostei moderadamente" e "Gostei regularmente.

Quanto a avaliação global do produto, utilizando a escala de "gostar ou desgostar", $95,84 \%$ dos avaliadores gostaram do produto e 2,08\% nem gostaram e nem desgostaram do produto, e 2,08 desgostaram ligeiramente (Figura 2).

Quanto a intenção de compra, apenas 2,08\% do público "Talvez compraria" (Figura 3), diferente do relatado por Dias et al. (2011) ao caracterizarem sensorialmente o licor de corte de maracujá constataram que $8 \%$ dos 50 provadores não comprariam o produto.

Figura 3. Avaliação comercial do licor de uva elaborado.

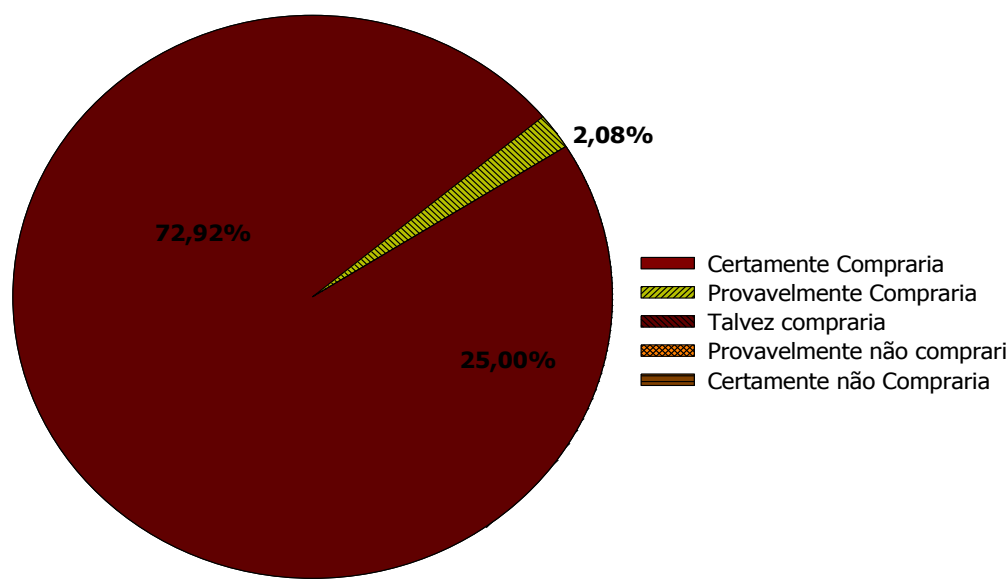

Os valores médios, das notas atribuídas pelos provadores para os atributos sensoriais sabor, cor, aroma e avaliação global, encontram-se na Tabela 1.

No teste de aceitação do licor todos os atributos obtiveram médias entre 8,46 a 8,66, variando entre gostei moderadamente e gostei extremamente. E para avaliação da intenção de compra, a média de 4,71 varia entre o certamente compraria e provavelmente comprariam ou seja, a formulação de licor desenvolvida apresentou nível de aceitação superior a 75\%, ou seja, mais de 3/4 dos entrevistados aprovaram o produto.

Em relação ao atributo cor, a formulação desenvolvida apresentou valores superiores aos relatados por Almeida et al. (2012) que obtiveram notas que variaram de 7,32 a 7,87 para este atributo, para licores de casca de tangerina, variedade Ponkan, e por Oliveira et al. (2014) que receberam notas entre 6,57 e 6,74 para sete formulações de licores de graviola. 
Tabela 1. Índice de aceitabilidade sensorial do licor de uva elaborado com a cv. 'Isabel'.

\begin{tabular}{ccc}
\hline Variável & Média & Índice de Aceitabilidade (\%) \\
\hline Cor & 8,66 & 96,30 \\
Aparência & 8,50 & 94,44 \\
Aroma & 8,46 & 93,98 \\
Sabor & 8,48 & 94,21 \\
Textura & 8,52 & 94,67 \\
Avaliação global & 8,44 & 93,75 \\
Intenção de compra & 4,71 & 94,17 \\
\hline
\end{tabular}

A cor foi a variável que apresentou maior aceitabilidade sensorial (Tabela 1). Segundo Oliveira et al. (2014), isso é justificando quando o licor apresenta coloração mais próxima a fruta in natura e maior viscosidade torna-se mais atrativos aos consumidores, ou seja, a formulação apresentou coloração característica de uva.

Dias et al. (2011) ao caracterizarem sensorialmente o licor de corte de maracujá amarelo obtiveram médias inferiores quando comparado ao licor de uva desenvolvido, variando de 6,6 a 7,3, nos atributos sabor, cor, aparência, consistência, aroma, e teor alcoólico, utilizando a mesma escala (Figura 1).

Segundo Teixeira et al. (1987) e Dutcosky (2011), para que um produto seja considerado como aceito, em termos de suas propriedades sensoriais, é necessário que obtenha um índice de aceitabilidade igual ou maior que $78 \%$. Considerando que o produto obteve um índice de aceitação superior a $92 \%$ (Tabela 1), conforme este autor este produto obteve uma ótima aceitação sensorial, ressalvando que não houve índices de "desgostar".

\section{CONCLUSÃO}

O licor de uva, elaborado a partir da cultivar 'Isabel' obteve excelente aceitabilidade sensorial pelos provadores, apresentando-se como uma opção para agregar valor a uva, bem como aumentando a diversidade de bebidas alcoólicas no mercado.

\section{AGRADECIMENTOS}

Ao Programa Nacional de Acesso ao Ensino Técnico e Emprego (PRONATEC) pela promoção do curso de Formação Inicial e Continuada em Produtores de Vinho e Derivados da Uva, executado através do Instituto Federal de Educação, Ciência e Tecnologia do Sertão Pernambucano, campus Petrolina Zona Rural.

\section{REFERÊNCIAS}

ABNT. Associação Brasileira de Normas Técnicas. NBR 14141: Escalas utilizadas em analise sensorial de alimentos e bebidas. Rio de Janeiro, 1998. 
ABREU, C. M.et al. Produtividade e qualidade da uva Isabel em função de doses de etefon e épocas de poda. Revista de Agricultura Neotropical, v. 4, p. 12-21, 2017.

ALBUQUERQUE, A. H. P.et al. Irrigação e fertirrigação potássica na cultura da videira em condições semiáridas. Pesquisa Agropecuária Tropical, v. 43, p 315-321, 2013.

ALMEIDA, E. L.et al. Elaboração de licor de casca de tangerina. Alimentos e Nutrição, v.23, p.259-265, 2012.

ANDRADE, V. P. M.et al. Yield and quality of 'Italia? grapes submitted to irrigation and fertilization control at the San Francisco Valley, Brazil. Comunicata Scientiae, v. 7, p. 175-182, 2016.

BARROS, J. C.et al. Obtenção e avaliação de licor de leite a partir de diferentes fontes alcoólicas. Global Science and Technology, v.1, p.27-33, 2008.

BECK, C.et al. Characterizing Global Climate Change by means of Köppen Climate Classification. Klimastatusbericht, p.139-149, 2005.

CALDAS, B. S.et al. Determinação de açúcares em suco concentrado e néctar de uva: comparativo empregando refratometria, espectrofotometria e cromatografia líquida. Scientia Chromatographica, v. 7, n. 1, p. 53-56, 2015.

CAMARGO, U. A.; MAIA, J. D. G.; RITSCHEL, P. Embrapa Uva e Vinho: novas cultivares brasileiras de uva. Bento Gonçalves, RS: Emprapa, 2010. 64 p.

DIAS, S. C.et al. Caracterização físico-química e sensorial do licor de corte do maracujá amarelo. Enciclopédia Biosfera, v.7, p.1405-1412, 2011.

DUTCOSKY, S. D. Análise sensorial de alimentos. 3.ed. Curitiba: Universitária Champagnat, 2011.426 p.

GULARTE, M. A. Análise sensorial. Pelotas: Universitária da Universidade Federal de Pelotas, 2009. $66 \mathrm{p}$.

MASCARENHAS, R. J.et al. Avaliação sensorial de uvas de mesa produzidas no Vale do São Francisco e comercializadas em João Pessoa-PB. Revista Brasileira de Fruticultura, Jaboticabal, v. 32, n. 4, p. 993$1000,2010$.

OLIVEIRA, E. N. A.et al.Estabilidade física e química de licores de graviola durante o armazenamento em condições ambientais. Revista Brasileira de Engenharia Agrícola e Ambiental, v. 19, p. 245-251, 2015.

OLIVEIRA, E. N. A. DE; SANTOS, D. C. Processamento e avaliação da qualidade de licor de açaí

(Euterpe oleracea Mart.). Revista do Instituto Adolfo Lutz, v. 70, p. 534-41, 2011.

OLIVEIRA, E.N.A.et al. Agroindustrial ulizaon of soursop (Annona muricata L.) for producon of liqueurs: Sensory evaluaon. Journal Of Biotechnology And Biodiversity. v.5, p. 33-42, 2014.

SEBRAE. Cachaça artesanal: relatóriocompleto. 2012. p. 81. (Série Estudos Mercadológicos).

SHAYANFAR, S.; BODBODAK, S. Effect of different physicochemical de-tartration methods on red grape juice quality. Journal of Food Science and Technology, v. 51, n. 12, p. 4084-4089, 2014.

SILVA, G. S.et al. Secagem e caracterização físico-química da Crimson. Blucher Chemical Engineerin Proceedings, v. 2, n. 1, p. 2071-2076, 2015.

SILVA, R. S.. Qualidade de uva 'Isabel' tratada com cloreto de cálcio em pós-colheita e armazenada sob atmosfera modificada. Revista Brasileira de Fruticultura, v. 34, p. 50-56, 2012. 
TEIXEIRA, E.et al. Análise sensorial de alimentos. Florianópolis: Ed. da UFSC, 1987. 180 p.

TEIXEIRA, L. J. Q.et al. Determinação da cinética de extração alcoólica no processamento de licor de café. Enciclopédia Biosfera, v. 6, n. 9, p. 1-9. 2010.

TEIXEIRA, L. J. Q.et al. Determinação da proporção de açúcar e fruta necessários para conferir os atributos ideiais ao licor de abacaxi. Enciclopédia Biosfera, v.8, p.1883-1889, 2012.

VIEIRA, V. B.et al. Produção, caracterização e aceitabilidade de licor de camu-camu (Myrciaria dubia (H.B.K.) McVaugh). Alimentos e Nutrição, v.21, p.519-522, 2010. 